\title{
INOCULACIÓN DE Brachiaria decumbens CON HONGOS FORMADORES DE MICORRIZA ARBUSCULAR NATIVOS DEL TRÓPICO HÚMEDO ECUATORIANO
}

\author{
${ }^{\bullet}$ Oscar Prieto Benavides ${ }^{1}$, Carlos Belezaca Pinargote ${ }^{2}$, Washington Mora Silva², Elías Vallejo Zambrano ${ }^{1}$, Víctor Gutiérrez \\ Lara $^{1}$, Edgar Pinargote Mendoza ${ }^{3}$. \\ ${ }^{1}$ Escuela de Ingeniería Forestal, Facultad de Ciencias Ambientales, Universidad Técnica Estatal de Quevedo, Campus \\ Ing. Manuel Haz Álvarez, km 1 11/2 vía a Santo Domingo de los Tsáchilas, C. P. 73. Quevedo, Los Ríos, Ecuador, \\ ${ }^{\circ}$ oscarprietob@hotmail.com \\ ${ }^{2}$ Unidad de Investigación Científica y Tecnológica, Facultad de Ciencias Ambientales, Escuela de Ingeniería Forestal, \\ Universidad Técnica Estatal de Quevedo. Campus Ing. Manuel Haz Alvarez, $\mathrm{km} 1$ 1/2 vía \\ a Santo Domingo de los Tsáchilas, C. P. 73. Quevedo, Los Ríos, Ecuador, \\ ${ }^{3}$ Departamento de Vinculación y Transferencia de Tecnologías, Facultad de Ciencias Pecuarias, \\ Universidad Técnica Estatal de Quevedo. Campus Ing. Manuel Haz Álvarez, $\mathrm{km} 1$ 1/2 vía \\ a Santo Domingo de los Tsáchilas, C. P. 73. Quevedo, Los Ríos, Ecuador,
}

\section{RESUMEN}

Q e realizó un estudio bajo condiciones semicontroladas de invernadero, para determinar el efecto de hongos formadores de micorriza arbuscular (HMA) nativos de sistema agroforestales tradicionales con Theobroma cacao L. (cacao) tipo nacional (SAF-C) en el Trópico húmedo ecuatoriano, sobre pasto Brachiaria decumbens. El experimento se realizó entre los meses junio y diciembre del año 2009, estuvo constituido por cinco tratamientos, que consistían en la inoculación de HMA originarios de SAF-C, distribuidos en un diseño completo al azar (DCA): T1: Glomus spp., T2: Scutellospora spp., T3: Glomus spp.+ Scutellospora spp., T4: Acaulospora spp. + Gigaspora spp., T5: Control $\left(\mathrm{H}_{2} \mathrm{O}\right.$ destilada estéril), en plántulas de $B$. decumbens sembradas en macetas plásticas de $1,000 \mathrm{~cm}^{3}$, conteniendo como sustrato una mezcla de suelo pobre en nutrientes + tamo de arroz, estériles, en proporción 3:1. El inóculo estuvo constituido de 30 esporas de HMA por tratamiento. Se analizaron las variables: a) número de esporas de HMA por $100 \mathrm{~g}$ de suelo húmedo $\left.\left(\mathrm{gsh}^{-1}\right), \mathrm{b}\right)$ porcentaje de colonización micorrícica visual y categoría de pelos radicales, c) altura de plantas, d) peso húmedo y seco del sistema foliar y radical, e) largo total de raíz (RL), y f) densidad radical (RLv), a 78 y 103 días después de las inoculaciones. Las plantas inoculadas con Glomus spp., o en combinación con Scutellospora spp. mostraron mejores respuestas en las variables evaluadas. Los resultados demostraron la eficiencia y potencial de los HMA procedentes de SAF-C, sobre plantas de B. decumbens.

Palabras claves: Trópico húmedo ecuatoriano, Glomus, Acaulospora, Gigaspora, Scutellospora, pelos radicales.
A study was conducted under semi-controlled greenhouse conditions to determine the effect of arbuscular mycorrhizal fungi (AMF) native of traditional agroforestry system with Theobroma cacao L. (cocoa) national type (SAF-C) in the ecuadorian humid Tropics, on Brachiaria decumbens grass. The experiment was carried between june and december of 2009, constituted by five treatments, involving the inoculation of AMF originating from AFS-C, distributed in a completely random design (CRD) as follows: T1: Glomus spp., T2 : Scutellospora spp., T3: Glomus spp. + Scutellospora spp., T4: Acaulospora spp. + Gigaspora spp., T5: Control (sterile distilled $\mathrm{H}_{2} \mathrm{O}$ ), in seedlings of $B$. decumbens planted in plastic pots of $1,000 \mathrm{~cm}^{3}$, containing as substrate a mixture of nutrient-poor soil + rice husk, steriles, in a 3:1 ratio. The inoculum was constituted by 30 spores of AMF per treatment. The variables analyzed were: a) number of spores of AMF by $100 \mathrm{~g}$ of moist soil $\left.\left(\mathrm{gms}^{-1}\right), \mathrm{b}\right)$ percentage of visual mycorrhizal colonization and root hairs category, c) plant height, d) wet and dry weight of leaf and root systems, e) total root length (RL) and f) root density (RLv) at 78 and 103 days after inoculation. Plants inoculated with Glomus spp. or in combination with Scutellospora spp. showed better responses in the variables measured. The results demonstrated the efficiency and potential of HMA from SAF-C, on plants of $B$. decumbens.

Key words: ecuadorian humid Tropics, Glomus, Acaulospora, Gigaspora, Scutellospora, root hairs.

Recibido: 21-Junio-2011. Recibido en forma corregida: 13-Septiembre2011. Aceptado: 8-Noviembre-2011.

Publicado como ARTÍCULO en Ciencia y Tecnología 4(2): 9-18. 2011 


\section{INTRODUCCIÓN}

$\mathrm{E}$ un hecho universalmente aceptado que las micorrizas estimulan el crecimiento, desarrollo y nutrición de las plantas, especialmente en suelos de baja y moderada fertilidad (Medina y Azcón, 2010). El papel de las micorrizas en la rizósfera de las plantas favorece la nutrición mineral, principalmente en cuatro aspectos: fisiología y desarrollo de la planta, crecimiento y morfología de raíces, procesos de absorción y disponibilidad de nutrimentos (Blanco y Salas, 1997; Medina y Azcón, 2010). Sin embargo, pese a que se conoce el potencial de las micorrizas arbusculares (MA), una apreciación cuantitativa de la contribución del hongo sobre la captación de nutrientes en ecosistemas naturales, presenta muchas dificultades, principalmente, porque son sistemas dinámicos (Borie et al., 2010), debido a esto, los estudios en su mayoría se centran a invernaderos o laboratorios, empleando especies vegetales fáciles de manipular, con resultados satisfactorios que reflejan de cerca la realidad de ecosistemas naturales. Desde el punto de vista nutricional de los vegetales, esta varía entre plantas anuales o perennes, siendo en el primer caso particularmente crítico el aporte de fósforo durante los primeros estados de crecimiento (Smith et al., 2003). Dados los resultados altamente satisfactorios en experiencias llevadas a cabo en invernadero, en la actualidad se están considerando grandes posibilidades de inoculación de hongos MA en campo, con el objetivo de mejorar el rendimiento de las cosechas agrícolas y disminuir la dependencia a los fertilizantes químicos tradicionales (Borie et al., 2010; Medina y Azcón, 2010).

Brachiaria decumbens es una gramínea perenne cultivada para la producción de forraje destinado a bovinos, se expande rápidamente, proporcionando una gran extensión de área verde, con una altura que varía de 40 a $90 \mathrm{~cm}$, es resistente al pisoteo, la quema y sequía. No obstante, las características naturales de $B$. decumbens han permitido que sean reconocidas por su gran facilidad de micorrización con hongos formadores MA (Combatt et al., 2008).

Considerando que existe la necesidad de investigar el potencial de hongos formadores de MA y su capacidad de infección en raíces de vegetales de fácil manejo, a ser empleados en futuros programas de producción de inoculantes micorrícicos para el Trópico Húmedo Ecuatoriano, en esta investigación se planteó como objetivos: a) evaluar la capacidad de micorrización radicular y producción de esporas de cuatro géneros de hongos MA, y b) su efecto sobre las variables agronómicas en $B$. decumbens, en un suelo de baja fertilidad, bajo condiciones semicontroladas.

\section{Materiales y Métodos}

\section{Fase de campo}

T a recolección de muestras de suelo para el Losterior aislamiento e identificación de los hongos formadores de micorriza arbuscular (MA) se realizó en sistemas agroforestales tradicionales con Theobroma cacao L. de tipo nacional (SAF-C), ubicados en fincas de la zona central del Trópico Húmedo Ecuatoriano, específicamente en la provincia de Los Ríos, cantones Quevedo y Valencia. Se consideraron SAF-C, que han sido conservados con escaso disturbio del suelo y sin aplicación de pesticidas químicos.

\section{Fase de Laboratorio}

La extracción de esporas de hongos MA desde muestras de suelo se realizó en el laboratorio de Microbiología Ambiental y Vegetal de la Universidad Técnica Estatal de Quevedo. Se empleó la metodología de tamizado y decantación en húmedo con centrifugación, propuesto por Gerderman y Nicholson (1963). Para la identificación de los hongos MA a nivel de género, se siguieron las claves taxonómicas e instrucciones propuestas por Brundrett et al. (1996); Powell y Bagyaraj (2000); Peterson et al. (2004); e INVAM (2009).

La evaluación del porcentaje de colonización micorrícica existente en las raíces de Brachiaria decumbens inoculadas, se realizó siguiendo la metodología de despigmentación y tinción de raíces, propuesta por Phillips y Hayman (1970) y Giovannetti y Mosse (1980) con modificaciones.

\section{Fase de Invernadero}

Como planta hospedera se utilizó la gramínea B. decumbens, sembrada en macetas de $1,000 \mathrm{~cm}^{3}$ de capacidad, conteniendo como sustrato una mezcla de suelo pobre en nutrientes + tamo de arroz, ambos estériles, en una proporción 3:1. Por cada maceta se sembraron tres plantas, que se inocularon a los 8 días después de la germinación con 30 esporas de hongos MA previamente aislados e identificados a nivel de género. Se realizaron riegos periódicos con $\mathrm{H}_{2} \mathrm{O}$ destilada estéril de acuerdo a la capacidad de campo del suelo (aproximadamente 200 $\mathrm{cm}^{3}$ cada 72 horas). El microclima formado dentro del invernadero permaneció a una temperatura aproximada de $25^{\circ} \mathrm{C}$ y $70 \%$ de humedad relativa. La evaluación de infección micorrícica y las variables agronómicas se realizaron en dos periodos, a los 78 y 103 días después de las inoculaciones. 


\section{Variables evaluadas}

Se evaluó el número de esporas por cada 100 $\mathrm{g}$ de suelo húmedo $\left(\mathrm{gsh}^{-1}\right)$, porcentaje de colonización micorrícica visual y categoría de pelos radicales, siguiendo la metodología y aplicando la escala propuesta por Herrera-Peraza et al. (2004) que se muestra en el cuadro 1. Las variables agronómicas evaluadas en las plantas de $B$. decumbens fueron: Altura de plantas $(\mathrm{cm})$, peso húmedo y seco del sistema foliar y radical $(\mathrm{g})$, largo total de raíz $(\mathrm{RL})(\mathrm{cm})$, densidad radical $(\mathrm{RLv})$ $\left(\mathrm{cm} \mathrm{cm}^{-3}\right)$. Toda la investigación se realizó en la época seca, entre los meses junio a diciembre del año 2009.

Cuadro 1. Escala empleada para la evaluación de densidad visual (\%) de micorrización y pelos radicales en raíces de Brachiaria decumbens. Propuesta por Herrera-Peraza et al. (2004)

\begin{tabular}{ccccccc}
\hline Categorías de micorrización & $\mathbf{0}$ & $\mathbf{1}$ & $\mathbf{2}$ & $\mathbf{3}$ & $\mathbf{4}$ & $\mathbf{5}$ \\
\hline Densidad visual (\%) & 0 & 1 & 2.5 & 15.5 & 35.5 & 47.5 \\
\hline
\end{tabular}

\begin{tabular}{ccccccc}
\hline Categorías pelos radicales & $\mathbf{0}$ & $\mathbf{1}$ & $\mathbf{2}$ & $\mathbf{3}$ & $\mathbf{4}$ & $\mathbf{5}$ \\
\hline Cantidad & Ausentes & Muy pocos & Pocos & Pocos & Muchos & Muchos \\
\hline \multirow{2}{*}{ Longitud } & & Cortos o & Cortos o & Cortos y & Cortos y \\
largos & largos & largos & largos & Largos \\
\hline
\end{tabular}

\section{Tratamientos y Diseño Experimental}

El experimento estuvo conformado por 5 tratamientos, cada uno constituido por un género de hongo MA: T1: Glomus spp., T2: Scutellospora spp., T3: Glomus spp. + Scutellospora spp., T4: Acaulospora spp. + Gigaspora spp., y T5: fue el control que consistió en la aplicación de $\mathrm{H}_{2} \mathrm{O}$ destilada estéril. Cada tratamiento contó con 10 repeticiones (macetas) distribuidas en un Diseño Completo al Azar. Las evaluaciones se realizaron en dos periodos, a 78 y 103 días después de las inoculaciones (cinco repeticiones por evaluación).

\section{Análisis Estadístico}

Se empleó el paquete estadístico SYSTAT 11 versión para Windows (Wass, 2004). Se efectuó un análisis de varianza ANOVA de una vía, y cuando existieron diferencias estadísticas significativas entre tratamientos se aplicó la prueba de separación de medias Tukey, testando probabilidades menores al 0.05 .

\section{Resultados y Discusión}

\section{Colonización micorrícica en raíces de $B$.} decumbens a nivel de invernadero.

$\mathrm{E}^{\mathrm{I}}$ análisis ANOVA mostró diferencias estadísticas significativas entre los tratamientos, tanto a los 78 días $(\mathrm{F}=86.5, \mathrm{p}=0.00)$ como a los 103 días $(\mathrm{F}=682.9, \mathrm{p}=0.00)$. En la figura 1, se muestran los valores promedio de densidad de colonización visual en raíces de $B$. decumbens, donde, a los 78 días, el tratamiento con Glomus spp., mostró el valor más alto de colonización micorrícica con respecto a los demás tratamientos, con una densidad visual del $2.6 \%$, seguido del tratamiento Glomus spp. + Scutellospora spp. con $1.6 \%$, aunque éste se mostró similar estadísticamente a los tratamientos Scutellospora spp. con el 1.2\% y Acaulospora spp. + Gigaspora spp. quien resultó ser el que menor colonización micorrícica presentó, con un valor de densidad visual de $0.8 \%$.

No obstante, a los 103 días se encontró una ligera disminución en los valores de densidad de colonización micorrícica visual en las raíces de $B$. decumbens en el tratamiento Glomus spp. con $2.1 \%$, mientras que los tratamientos Glomus spp. + Scutellospora spp. y Scutellospora spp., mostraron un leve aumento con valores de $1.7 \%$ y $1.2 \%$ respectivamente. En lo que se refiere al tratamiento Acaulospora spp.+Gigaspora spp. no se encontró aumento en su porcentaje de colonización con respecto a la evaluación anterior y se mantuvo en un $0.8 \%$ de densidad visual. Con respecto al tratamiento control $\left(\mathrm{H}_{2} \mathrm{O}\right.$ destilada estéril), este, como era de esperarse, no presentó colonización micorrícica en ninguno de los periodos evaluados.

Los valores de colonización micorrícica visual obtenidos en raíces de $B$. decumbens a los 78 y 103 días después de inoculadas, contrastan ligeramente con los encontrados por otros investigadores, como Vázquez et al. (2010), quienes utilizando como planta hospedera la misma especie forrajera, encontraron densidades de colonización micorrícica visual cercanos a los alcanzados en la presente investigación en tres 
periodos de evaluación 60, 90, y 120 días después de las inoculaciones, y además demuestra que pasados los 100 días, la colonización micorrícica visual disminuye considerablemente en plantas inoculadas con especies fúngicas del género Glomus. No obstante, diferencias mayores fueron encontradas al comparar los estudios realizados por Ben et al. (2003), quienes buscando respuestas fisiológicas de Trifolium alexandrinum L., mediante la inoculación de consorcios micorrícicos, obtuvieron tasas de micorrización superiores al 70\% a los 60 días después de las inoculaciones. Por otro lado, Jia et al. (2004) también obtuvieron elevados porcentajes de micorrización radicular en Vicia faba a los 54 y 63 días después de las inoculaciones. Además en un ensayo con Medicago sativa L., (alfalfa), Irrazabal et al. (2004) detectaron porcentajes altos de micorrización a partir de los 30 días después de la siembra. No obstante, cabe señalar que los investigadores antes señalados no utilizaron como inóculo únicamente esporas de hongos micorrícicos identificados previamente, sino que en su defecto emplearon diferentes consorcios micorrícicos de distintos géneros, lo cual pudo favorecer el incremento de micorrización en las plantas, ya que de esta forma es difícil precisar (número desconocido) la cantidad de esporas, la cantidad de micelio extraradical, o los segmentos de raíces colonizadas que lleva el inóculo. Reflexionando, podría deducirse que el inóculo empleado por estos investigadores contendría elevadas cantidades de unidades de infección y por lo tanto, existía una mayor probabilidad de aumento en el porcentaje de infección.

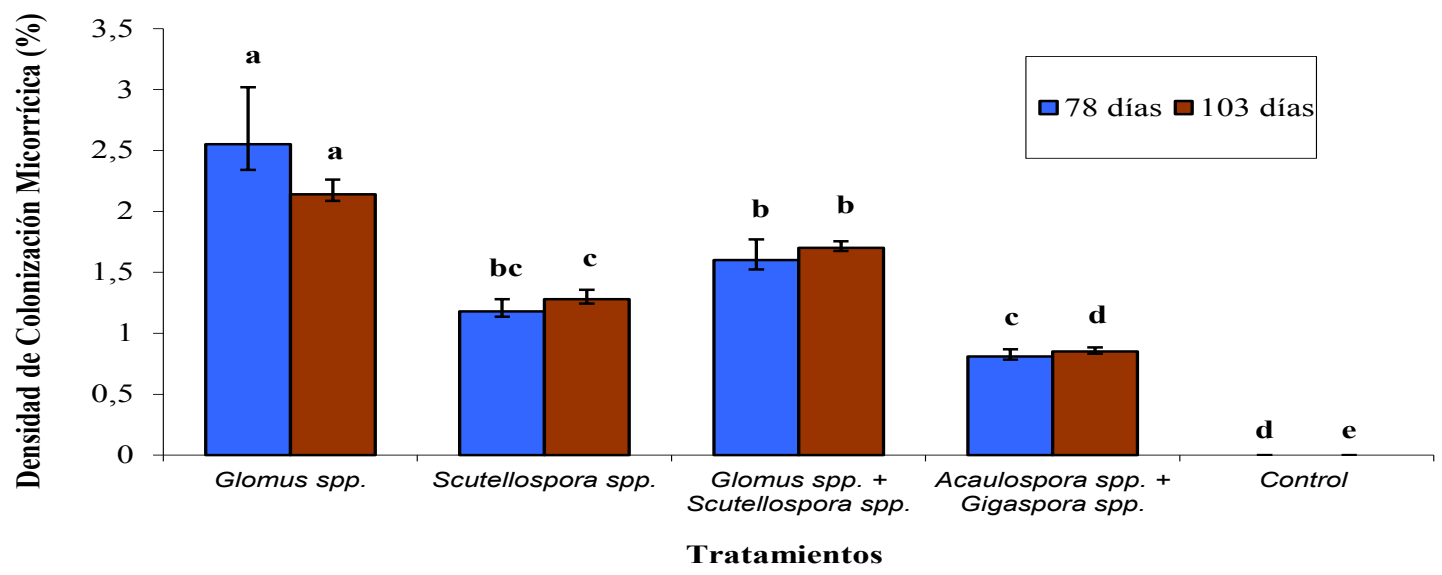

Figura 1. Densidad de colonización micorrícica visual en raíces de $B$. decumbens bajo cinco tratamientos a los 78 y 103 días después de las inoculaciones. Los valores representan la media de 5 repeticiones, con barras de desviación estándar y error estándar. Medias seguidas por la misma letra no presentan diferencias estadísticas significativas ( $\mathbf{p} \leq 0.05)$. UTEQ. 2011.

2. Densidad de esporas por volumen de suelo en sustrato a nivel de invernadero.

En la figura 2, se muestra la densidad de esporas de hongos MA por volumen de suelo, tomando como referencia $100 \mathrm{~g}$ de suelo húmedo $\left(\mathrm{gsh}^{-1}\right)$. El análisis ANOVA permitió determinar la existencia de diferencias estadísticas significativas entre tratamientos, tanto a los 78 días $(\mathrm{F}=109.8, \mathrm{p}=0.00)$ y 103 días $(\mathrm{F}=285.9$, $\mathrm{p}=0.00$ ) después de las inoculaciones. A los 78 días, el tratamiento Glomus spp. mostró la mayor concentración con 306 esporas, aunque con similitud estadística frente al tratamiento Glomus spp. + Scutellospora spp., que presentó 275 esporas, mientras que los tratamientos Scutellospora spp. y Acaulospora spp. + Gigaspora spp. con 58 y 17 esporas respectivamente se comportaron muy por debajo de los tratamientos expuestos anteriormente. No obstante, en el tratamiento control ( $\mathrm{H}_{2} \mathrm{O}$ destilada estéril), como era de esperarse, no se encontraron esporas. A los 103 días la tendencia existente entre los tratamientos se mantuvo, dando como resultado un incremento en el número de esporas para todos los tratamientos, excepto en el tratamiento control $\left(\mathrm{H}_{2} \mathrm{O}\right.$ destilada estéril). Los tratamientos Glomus spp. y Glomus spp. + Scutellospora spp. mostraron medias de 343 y 292 esporas, mientras que los tratamientos Scutellospora spp. y Acaulospora spp. + Gigaspora spp. alcanzaron 71 y 29 esporas en su orden, y siguieron siendo los más bajos en relación a los primeros.

Investigaciones realizadas en otras latitudes muestran comportamientos distintos en hongos formadores de MA, en lo referente a su fisiología, en este sentido, Castillo et al. (2006) encontró densidades superiores a los valores expuestos en la presente investigación, tomando como referencia a especies del género Glomus, obteniendo a los 120 días, promedios 


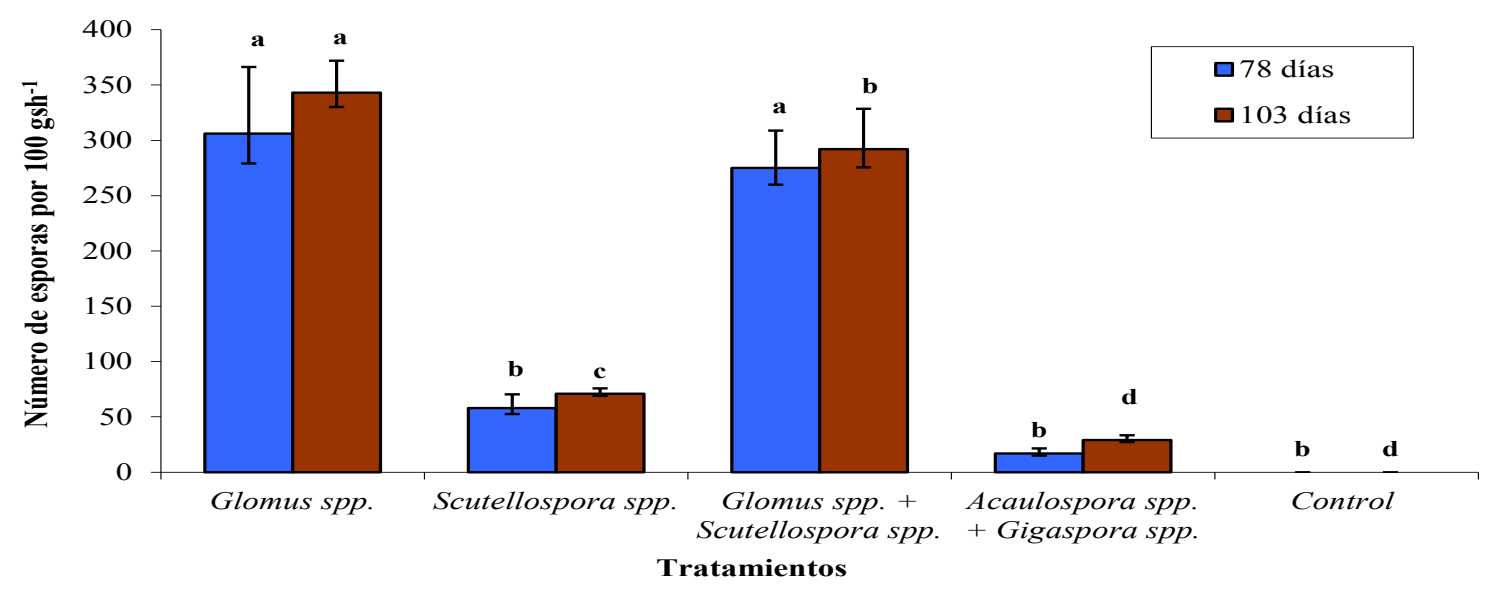

Figura 2. Densidad de esporas de hongos formadores de MA en $100 \mathrm{gsh}^{-1} \mathrm{bajo}$ plantas de $B$. decumbens en condiciones de invernadero a los 78 y 103 días después de las inoculaciones. Los valores representan la media de cinco repeticiones, con barras de desviación estándar y error estándar. Medias seguidas por la misma letra no presentan diferencias estadísticas significativas $(\mathbf{p} \leq \mathbf{0 . 0 5})$. UTEQ. 2011.

de 2,500 esporas de Glomus claroideum y 2,000 de Glomus etunicatun por cada $100 \mathrm{~g}$ de suelo, inoculados previamente en Trifolium aestivum y Avena sativa L. No obstante, en la investigación antes señalada se utilizó como inóculo consorcios micorrícicos constituidos por esporas, micelio extraradical y trozos de raíces micorrizadas de las mencionadas especies, lo cual pudo favorecer el incremento de esporulación. Además, el número de días desde la inoculación hasta la evaluación final fue mayor al empleado en esta investigación, lo cual hace suponer que el tiempo sería un factor determinante con respecto a esta variable. Otro factor que posiblemente afectó la esporulación sería la especie vegetal utilizada como hospedante, ya que algunas son más susceptibles a la micorrización que otras. Por otra parte el estrés hídrico al que se someten las plantas inoculadas antes de su evaluación, influye positivamente en el incremento de la densidad de esporas en el sustrato, tal como lo mencionan Guzmán-Gonzáles y Farías-Larios (2005) que en su estudio sobre la biología y regulación molecular de la micorriza arbuscular, demostraron que al someter plantas hospedadoras a estrés hídrico durante tres semanas antes de su evaluación, la esporulación se incrementa en un $30 \%$ con respecto a las plantas no sometidas a estrés.

3. Categoría de pelos radicales en raíces de Brachiaria decumbens

Para los dos periodos se obtuvieron diferencias estadísticas significativas entre los tratamientos. A los 78 días $(\mathrm{F}=6.78, \mathrm{p}=0.001)$, las plantas inoculadas con el tratamiento Glomus spp. mostraron la mayor cantidad de pelos radicales con respecto a los demás tratamientos, con una densidad de 2.7 , seguido del tratamiento Glomus spp. + Scutellospora spp. con 2.4. Los tratamientos Scutellospora spp. y Acaulospora spp. + Gigaspora spp. mostraron valores de 2.3 y 2.2 , respectivamente, resultaron estadísticamente similares al tratamiento control $\left(\mathrm{H}_{2} \mathrm{O}\right.$ destilada estéril), que presentó una categoría de pelos radicales de 2 , siendo el que obtuvo la menor densidad de pelos radicales. Mientras que a los 103 días $(\mathrm{F}=7.67, \mathrm{p}=0.001)$, los tratamientos Glomus spp.; Glomus spp. + Scutellospora spp.; Scutellospora spp. y Acaulospora spp.+ Gigaspora spp., obtuvieron valores idénticos de 2.8 de densidad de pelos radicales y no presentaron diferencias estadísticas entre sí, sin embargo, fueron estadísticamente distintos al tratamiento control $\left(\mathrm{H}_{2} \mathrm{O}\right.$ destilada estéril) que obtuvo un valor 2.1 (Figura 3 ).

Los resultados obtenidos con respecto a esta variable, demuestran que la inoculación inducida de hongos micorrícicos arbusculares en plantas de $B$. decumbens estimula el desarrollo e incremento del número de pelos radicales, independientemente que el inóculo este constituido por uno o varios géneros fúngicos formadores de MA. Capacidad que brinda a las plantas ventajas comparativas frente a la exploración de nutrientes y agua en la rizósfera.

\section{Altura de plantas de Brachiaria decumbens}

En la evaluación realizada a los 78 días después de las inoculaciones, no se obtuvieron diferencias estadísticas significativas $(\mathrm{F}=0.328, \mathrm{p}=0.856)$, mientras que a los 103 días se encontraron diferencias estadísticas 


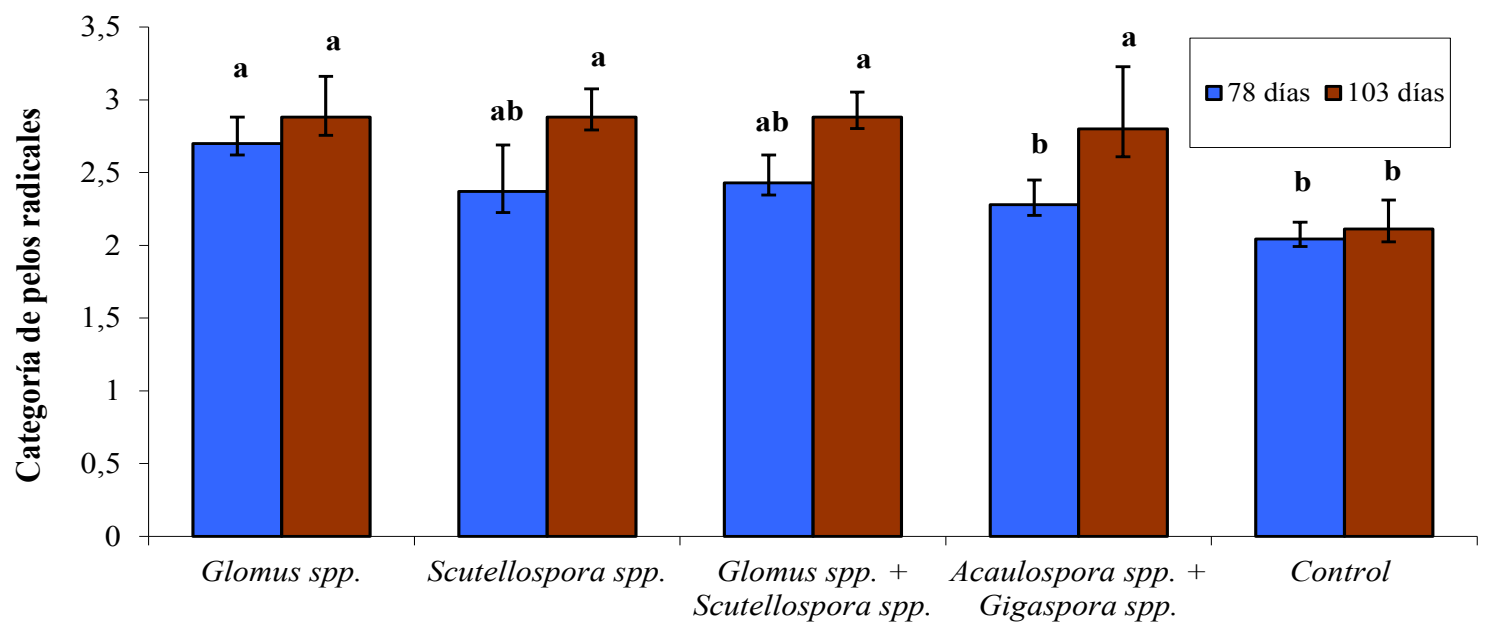

Tratamientos

Figura 3. Categoría de pelos radicales en plantas de B. decumbens inoculadas con hongos formadores de MA, a los 78 y 103 días después de inoculadas. Los valores representan la media de 5 repeticiones, con barras de desviación estándar y error estándar. Medias seguidas por la misma letra no presentan diferencias estadísticas significativas ( $\mathbf{p} \leq 0.05)$. UTEQ. 2011.

entre tratamientos $(\mathrm{F}=8.935, \mathrm{p}=0.000)$, donde las plantas inoculadas con Glomus spp. y Glomus spp. + Scutellospora spp., presentaron la mayor altura, con 132.5 y $127.7 \mathrm{~cm}$ respectivamente, mostrando estos tratamientos un comportamiento parecido al de las plantas inoculadas con Acaulospora spp. + Gigaspora spp., mientras que los tratamientos Scutellospora spp. y control $\left(\mathrm{H}_{2} \mathrm{O}\right.$ destilada estéril), fueron los que menor altura alcanzaron (Cuadro 2).

Los resultados obtenidos en esta investigación son parecidos a los reportados por Díaz et al. (2008), quienes utilizando la gramínea Sorghum bicolor L. (sorgo) como planta hospedera y empleando como inóculo un consorcio micorrícico de Glomus intraradices, tampoco encontraron diferencias estadísticas significativas en su primera evaluación a los 60 días. Sin embargo, en la segunda evaluación realizada a los 90 días, las plantas de sorgo mostraron diferencias estadísticas significativas, obteniendo alturas promedio de $120 \mathrm{~cm}$, frente a $97 \mathrm{~cm}$ que presentaron plantas sin inoculación previa. Esto demuestra la importancia de los hongos formadores de MA, en especial del género Glomus para estimular el crecimiento de plantas, situación que se refleja al compararlas a nivel de laboratorio con plantas carentes de inoculación inducida con hongos MA, independientemente de la planta utilizada como hospedante.

De los géneros de hongos formadores de MA, aislados desde Sistemas Agroforestales tradicionales con Theobroma cacao L. (cacao) tipo nacional (SAF-C) y posteriormente inoculados en plantas de $B$. decumbens, el género Glomus principalmente, ha sido estudiado ampliamente y utilizado como inoculante para mejorar el desarrollo en distintas especies vegetales. Sin embargo, poco se conoce de los demás géneros fúngicos estudiados en esta investigación, especialmente en lo relacionado a beneficios nutricionales específicos hacia diferentes especies vegetales. No obstante, se ha generalizado y aceptado universalmente que los hongos formadores de MA tienen la capacidad de estimular y aumentar el desarrollo vegetal, sin desconocer cierta especificidad y variabilidad genética, que hace de algunas especies fúngicas más efectivas que otras, y aceptando que en sí, todas las especies de hongos micorrícicos en mayor o menor grado pueden hacerlo (Brundrett et al., 1996; Klironomos, 2003).

\section{Peso húmedo y seco del sistema foliar y radical}

El análisis ANOVA no mostró diferencias estadísticas significativas para el peso húmedo $(\mathrm{F}=0.755, \mathrm{p}=0.566)$ y seco $(\mathrm{F}=0.568, \mathrm{p}=0.689)$ del sistema foliar a los 78 días después de las inoculaciones. Sin embargo, a los 103 días se obtuvieron diferencias estadísticas significativas entre los tratamientos, tanto para el peso húmedo $(\mathrm{F}=3.972, \mathrm{p}=0.016)$ como para el peso seco $(\mathrm{F}=3.306, \mathrm{p}=0.031)$. Los tratamientos cuyas plantas fueron inoculadas con hongos de MA presentaron valores de peso estadísticamente superiores al tratamiento control $\left(\mathrm{H}_{2} \mathrm{O}\right.$ destilada estéril). No obstante, los resultados obtenidos son comparables a los alcanzados por algunos grupos de investigadores 
como González et al. (2008), y a la vez contrastan con los logrados en otros trabajos. En este sentido, Sosa et al. (2006) al inocular plantas de B. decumbens con 20 esporas pertenecientes a una mezcla de los géneros Glomus y Acaulospora, alcanzaron promedios de peso seco del sistema foliar y radicular muy por debajo de los obtenidos en el presente estudio, con $0.054 \mathrm{~g}$ planta $^{-1}$ y $0.013 \mathrm{~g}$ planta $^{-1}$ respectivamente, mientras que las plantas carentes de inoculación redujeron su peso considerablemente a los 76 días después de las inoculaciones. Por otro lado, Plana et al. (2008), inoculando un coctel de hongos formadores de micorriza arbuscular en $B$. decumbens como planta hospedera, obtuvo mejores resultados a los encontrados en la presente investigación, con pesos superiores en plantas inoculadas frente a las sin inocular. No obstante, es necesario señalar que las condiciones, el contenido del inóculo, así como los sustratos utilizados por los autores arriba mencionados fueron diferentes a los empleados en la presente investigación, por lo cual se puede atribuir a estos factores la variación de resultados, aunque sí confirman que los hongos formadores de MA aumentan la masa foliar, tanto en peso húmedo como en peso seco, independiente de cómo este constituido el inóculo a utilizarse (Cuadro 2).

En lo referente al peso húmedo y seco del sistema radical por planta, el análisis ANOVA no mostró diferencias estadísticas significativas a los 78 días $(\mathrm{F}=0.595, \mathrm{p}=0.671 ; \mathrm{F}=0.662, \mathrm{p}=0.625)$ ni a los 103 días $(\mathrm{F}=2.509, \mathrm{p}=0.068 ; \mathrm{F}=3.770, \mathrm{p}=0.078)$ respectivamente (Cuadro 2).

\section{Longitud total de raíz por planta (RL) y Longitud de raíz por volumen de suelo (RLv)}

Para la variable RL, el análisis ANOVA no determinó diferencias estadísticas significativas entre los tratamientos a los 78 días después de las inoculaciones $(\mathrm{F}=0.386, \mathrm{p}=0.816)$, mientras que a los 103, las diferencias estadísticas fueron detectadas $(\mathrm{F}=4.779, \mathrm{p}=0.007)$. Para este periodo, el tratamiento Glomus spp. + Scutellospora spp., con $169.6 \mathrm{~cm}$ de longitud del sistema radicular por planta, mostró ser superior a los demás, mientras que el tratamiento control ( $\mathrm{H}_{2} \mathrm{O}$ destilada estéril) con $103.7 \mathrm{~cm}$ fue el que menor longitud mostró (Cuadro 2).

Paralelamente, la variable RLv reflejó diferencias estadísticas significativas entre los tratamientos a los 78 días $(\mathrm{F}=5.337, \mathrm{p}=0.004)$ y 103 días $(\mathrm{F}=26.431, \mathrm{p}=0.000)$ después de las inoculaciones. A los 78 días, los tratamientos Glomus spp., Glomus spp.+ Scutellospora spp., y Scutellospora spp. fueron estadísticamente superiores a los demás tratamientos, obteniendo $0.25,0.24$ y $0.23 \mathrm{~cm} \mathrm{~cm}^{-3}$ respectivamente, frente al tratamiento control $\left(\mathrm{H}_{2} \mathrm{O}\right.$ destilada estéril) quien mostró la menor longitud de raíz por volumen de suelo explorado $\left(0.18 \mathrm{~cm} \mathrm{~cm}^{-3}\right)$. Mientras que a los 103 días, los tratamientos Glomus spp. y Glomus spp. + Scutellospora spp. con 0.37 y $0.34 \mathrm{~cm} \mathrm{~cm}^{-3}$ respectivamente, siguieron siendo estadísticamente superiores con relación a los demás tratamientos (Cuadro 2).

La habilidad de las plantas para sacar ventaja de los nutrientes disponibles en el suelo, está sujeta a las propiedades morfológicas y fisiológicas de su sistema radicular, donde, las variables longitud total de raíz por planta $(\mathrm{RL})$ y longitud de raíz por volumen de suelo, o también conocida como densidad de longitud radicular $(\mathrm{RLv})$ son parámetros que caracterizan la capacidad de absorción de una especie vegetal (Jungk y Claassen, 1997). De acuerdo a los resultados obtenidos en la presente investigación, parece existir una relación positiva entre la presencia de hongos formadores de MA y el incremento en la densidad de su sistema radicular. En este sentido, la simbiosis raíz-hongo micorrícico, estimuló el incremento en las variables RL y RLv, debido posiblemente a la ventaja que las raíces micorrizadas poseen para explorar mayor volumen de suelo y compensar sus necesidades nutricionales captando especialmente aquellos nutrientes de poca movilidad como el fósforo (Kovar y Claassen, 2005). Investigaciones realizadas por Moreno (1988) en Solanum tuberosum (papa) empleando consorcios micorrícicos de $G$. fasciculatum, confirman este hecho, donde demostró que plantas micorrizadas presentaban una mayor de nsidad radicular que plantas no micorrizadas.

Los resultados hacen sospechar que hongos formadores de MA, y en especial las especies del género Glomus estimulan en las plantas incrementar su sistema radicular ( $R L)$ y densidad radicular (RLv). Esto se debe a que las especies del género Glomus se reproducen con mayor rapidez que especies de otros géneros como Scutellospora, Acaulospora o Gigaspora, y también desarrollan mayor cantidad de hifas, que a su vez incrementa la capacidad de infección (Irrazabal et al., 2004).

\section{Conclusiones}

$\mathrm{L}$ a eficiencia de hongos formadores de MA en la infección radicular y su contribución para mejorar la calidad de plantas de $B$. decumbens, demuestran los beneficios fisiológicos y ecológicos ejercidos por estos microorganismos.

La respuesta positiva ejercida por hongos formadores de MA originarios de sistemas agroforestales con $T$. cacao L. sobre B. decumbens, crea la necesidad 
Prieto et al.

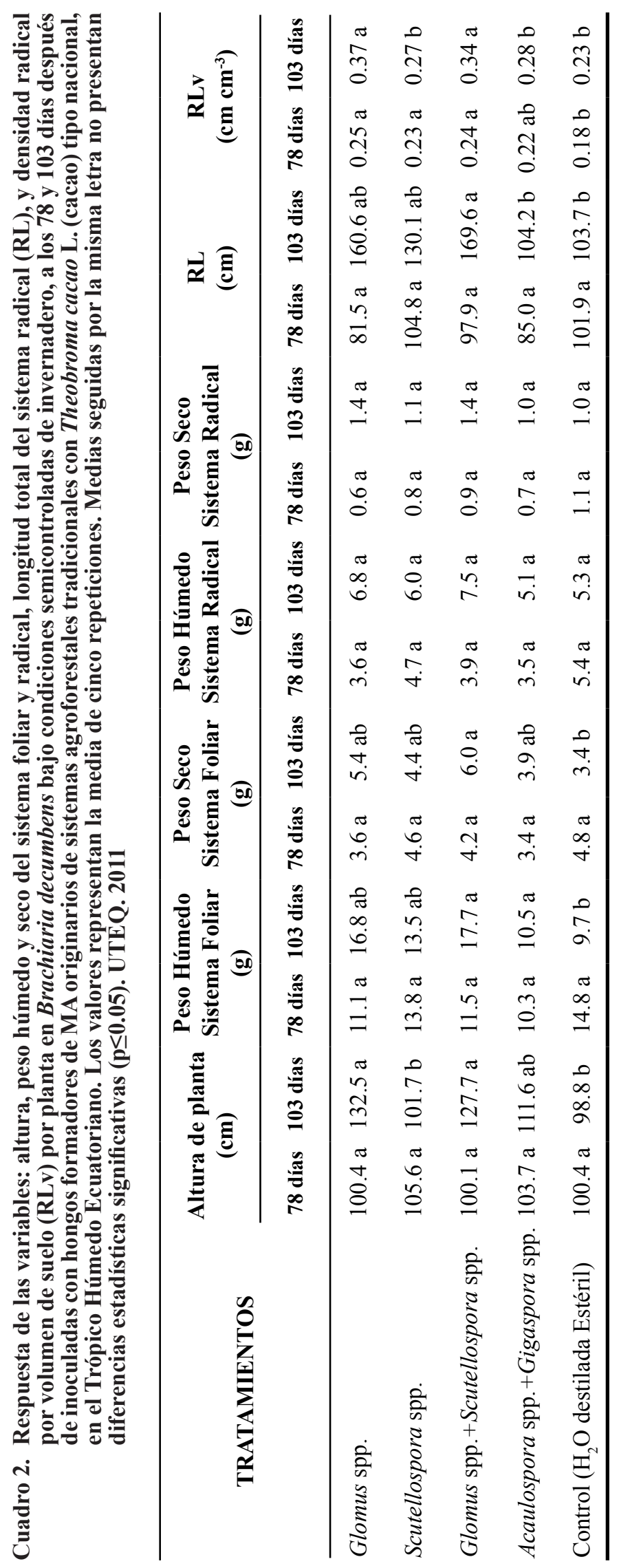


de contar con un banco germoplásmico ex situ en el Trópico Húmedo Ecuatoriano y seleccionar hongos eficientes destinados a la producción de inoculantes para especies agrícolas y forestales de importancia económica.

Por último, los sistemas agroforestales con T. cacao, tradicionales en el Trópico Húmedo Ecuatoriano, deben ser conservados, ya que guardan una valiosa microfauna, destacándose entre ella los hongos formadores de micorriza arbuscular.

\section{LiTERATURA CITADA}

Ben, L., A. Morte, E. Ouarraqi y A. Oihabi. 2003. Réponses physiologiques et biochimiques dú tréfle (Trifolium alexandrinum L.) a la double association Mycorrhizes-Rhizobium sous une contrainte saline. Agronómic 23:571-580.

Blanco, F. y E. Salas. 1997. Micorrizas en la Agricultura: Contexto mundial e investigación realizada en Costa Rica. Agronomía Costarricense 21(1):5567.

Borie, F., R. Rubio, A. Morales, G. Curaqueo and P. Cornejo. 2010. Arbuscular mycorrhizae in agricultural and forest ecosystems in Chile. J. Soil. Sci. Plant Nutr. 10(3):185-206.

Brundrett, M., N. Bougher, B. Dell, T. Grove and N. Malajczuk. 1996. Working with mycorrhizas in forestry and agriculture. ACIAR Monograph. Camberra, Australia. 374 p.

Castillo, C, G., F. R. Borie, R. E. Godoy, R. E. Rubio and E. Sieverding. 2006. Diversity of mycorrhizal plant species and arbuscular mycorrhizal fungi in evergreen forest, deciduous forest and grassland ecosystems of southern Chile. Journal of Applied Botany and Food Quality 81(1):40-47.

Combatt, E., A, Jarma y L. Maza. 2008. Crecimiento de Brachiaria decumbens Stapf y Cynodon nlemfuensis Vanderyst en suelos sulfatados ácidos de córdoba. Rev. MVZ. Córdoba, 13(2):13801392.

Díaz, A., C. Jacques y M. Peña. 2008. Productividad de sorgo en campo asociada a micorriza arbuscular y Azospirrillum brasilense. Villahermosa, MX. Universidad y Ciencia 24:227-234.

Gerderman, J., and T. Nicholson. 1963. Spores of mycorrhizal endogene species extracted from soil by wet sieving and decanting. Transactions of the British Mycological Society 46:235-244.

Giovannetti, M. and B. Mosse. 1980. An evaluation of techniques for measuring vesicular-arbuscular infection in roots. New phytologist 84:489-500.

González, P. J., R. Plana, R. Rivera, F. Fernández y J. Arbola. 2008. Efectos de la inoculación de hongos micorrízicos arbusculares en pastos del género Brachiaria, cultivados en suelo Pardo Mullido. Revista Cubana de Ciencia Agrícola 42(1):101106.

Guzmán-González, S. y J. Farías-Larios. 2005. Biología y regulación molecular de la micorriza arbuscular. Avances en investigación agropecuaria 9(2):1731.

Herrera-Peraza, R. A., E. Furrazola, Y. Torres-Arias. 2004. Functional strategies of root hairs and arbuscular mycorrhizae in an evergreen tropical forest, Sierra del Rosario, Cuba. Revista Cenic; Ciencias Biológicas (La Habana), 35(2):113-123.

INVAM (International Culture Collection of Vesicular Arbuscular Mycorrhizal Fungi). 2009. Identification to Genus (en línea). Consultado 15 Aug. 2009. Disponible en http://invam.caf.wvu. edu/fungi/taxonomy/genuskey.htm

Irrazabal, G., S. Velazquez y M. Cabello. 2004. Efectividad y diversidad de hongos micorrízicos arbusculares de la rizósfera de los talares de Magdalena, Provincia de Buenos Aires, AR. Boletín Micológico 19:49-57.

Jia, Y., V. Gray and C. Straker. 2004. The influence of Rhizobium and Arbuscular Mycorrhizal fungi on nitrogen and phosphorus accumulation by Vicia faba. Annals of Botany 94:251-258.

Jungk, A. and N. Claassen. 1997. Ion diffusion in the soil-root system. Advances in Agronomy 61:53110.

Klironomos, J. N. 2003. Variation in plant response to native and exotic arbuscular mycorrhizal fungi. Ecology 84:2292-2301.

Kovar, J. and N. Claassen. 2005. Soil-root interactions and phosphorus nutrition of plants. In: Sims, J. and Sharpley, A. (eds) Phosphorus: Agriculture and the environment. ASA, CSSA, and SSSA, Madison, WI. Agronomy Monographs 46:379414.

Medina, A. y R. Azcón. 2010. Effectiveness of the application of arbuscular mycorrhizal fungi and organic amendments to improve soil quality and plant performance under stress conditions. j. soil sci. plant nutr. 10(3):354-372.

Moreno, P. 1988. Inoculación de micorrizas MVA en papa (Solanum tuberosum) respuesta en el crecimiento y nutrición de plantas inoculadas en invernadero y en campo. Revista Latinoamericana de la Papa 1:84-103.

Peterson, R., H. Massicotte and L. Melvilla. 2004. Mycorrhizas: Anatomy and Cell Biology. Otawa, CAN. NRC, Research Press. 173 p.

Phillips, J. and D. Hayman. 1970. Improved procedures for clearing roots and staining parasitic and 
vasicular-arbuscular mycorrhizal fungi for rapid assessment of infection. Transactions of the British Mycological Society 55:158-161.

Plana, R., R. Rivera, F. Fernández, J. Arbola. 2008. Efecto de la inoculacion de hongos micorrizicos arbusculares en pastos del género Brachiaria, cultivados en suelo pardo mullido. Revista Cubana de Ciencia Agrícola 42(1):101-106.

Powell, C. and D. Bagyaraj. 2000. MA Mycorrhiza. Florida. Printed in the United States. p 232.

Smith, S., F. Smith and I. Jakobsen. 2003. Mycorrhizal fungi can dominate phosphate supply to plants irrespective of growth responses. Plant Physiology 130:16-20.

Sosa, T., J. Sánchez, E. Morales, F. Cruz. 2006. interacción Micorrizas Arbusculares- Trichoderma harzianum (Moniliaceae) y efectos sobre el crecimiento de Brachiaria decumbens (Poaceae). Acta Biológica Colombiana 11(1):43-54.
Vázquez, B., R. Rivera, K. Fernández y Y. Rodríguez. 2010. Caracterización del comportamiento micorrízico en Brachiaria decumbens inoculada con Glomus hoi-like. Cultivos Tropicales 31(3): 21-26

Wass, J. 2004. Systat. More Graphs, Less Effort: Integrated desktop statistics and graphics package features many improvements. User's manual. Systat Software. San Jose, USA. 416 p. 